\title{
Some Third Order Methods for Solving Nonlinear Equations
}

\author{
Jivandhar Jnawali \\ Department of Mathematics, Ratna Rajyalaxmi Campus, Tribhuvan University, Nepal \\ Corresponding author: jnawalij@gmail.com
}

Received: March 15, 2017 Revised: May 30, $2017 \quad$ Accepted: June 5, 2017

\begin{abstract}
There are several third order numerical methods having same efficiency index appeared in literature for solving nonlinear equations of a single variable. Practically, if we apply these methods in different nonlinear equations, we can observe that all methods are not performed equally for given nonlinear equations. The main objective of this paper is to show through numerical experiment that the performance of some third order methods having the same efficiency index does not perform equally for particular nonlinear equation. For the numerical comparison, we use Matlab software.
\end{abstract}

2010 AMS Subject Classification: 65H05

Keywords: Newton method, nonlinear equations, order of convergence, contra harmonic mean

\section{Introduction}

Solving nonlinear equations of single variable is one of the most important problems in numerical analysis. Nonlinear equations have appeared in almost every branches of science and engineering. Finding the solution of this type of equations analytically is almost impossible. So several researchers are interested to find the numerical solution of nonlinear equations. During last two decades, a lot of new efficient numerical methods have been obtained by several authors for solving nonlinear equations. One of the well-known and widely used numerical method for solving nonlinear equation $f(x)=0$, where $f: D \subset R \rightarrow R$ a scalar function on an open interval $D$, is the Newton method [2]

$$
x_{n+1}=x_{n}-\frac{f\left(x_{n}\right)}{f^{\prime}\left(x_{n}\right)},
$$

which converges quadratically to simple root $\alpha$ of $f$, i.e., $f(\alpha)=0$ and $f^{\prime}(\alpha) \neq 0$. In the literature, several variants of this method have appeared for solving nonlinear equations with higher order of convergence. Some of them can be found in [1, 4-13].

Definition: [3] Let $\left\{x_{n}\right\}$ be a sequence of iterates of some numerical method to solve a nonlinear equation $f(x)=0$ and $e_{n}$ be the error in $x_{n}$, i.e., $x_{n}=\alpha+e_{n}, \alpha$ being an exact 
root of $f(x)=0$. The numerical method is said to be of order $p$ if $p$ is the smallest positive number such that there exists a constant $A$ and

$$
e_{n+1}=A e_{n}{ }^{p} \quad \forall \quad n=1,2,3, \ldots
$$

This equation is generally known as the error equation for used numerical method.

Definition: [9] Efficiency index is simply defined as $p^{\frac{1}{m}}$, where $p$ is the order of convergence of the method and $\mathrm{m}$ is the number of function evaluations required by the method per iteration. The efficiency index of Newton method is 1.41 .

\section{Description of Methods}

Suppose $\alpha$ be a simple zero of a sufficiently differentiable function $f$ and consider the numerical solution of the equation $f(x)=0$. Weerakoon and Fernando [13] used the technique of numerical integration to improve Newton's method. In fact, they approximated the integral in the Newton's theorem

$$
f(x)=f\left(x_{n}\right)+\int_{x_{n}}^{x} f^{\prime}(t) d t
$$

by trapezoidal rule, that is,

$$
\int_{x_{n}}^{x} f^{\prime}(t) d t=\frac{x-x_{n}}{2}\left[f^{\prime}\left(x_{n}\right)+f^{\prime}(x)\right]
$$

and then obtained the following third order method as a variant of Newton's method:

$$
\begin{array}{ll} 
& x_{n+1}=x_{n}-\frac{2 f\left(x_{n}\right)}{f^{\prime}\left(x_{n}\right)+f^{\prime}\left(x_{n}^{*}\right)} \\
\text { where } & x_{n}^{*}=x_{n}-\frac{f\left(x_{n}\right)}{f^{\prime}\left(x_{n}\right)} .
\end{array}
$$

The method (3) is known as arithmetic mean Newton's method, which can also be expressed as

$$
x_{n+1}=x_{n}-\frac{f\left(x_{n}\right)}{\frac{f^{\prime}\left(x_{n}\right)+f^{\prime}\left(x_{n}^{*}\right)}{2}}
$$

If we compare the method (4) with the Newton's method (1), the difference is only we replace $f^{\prime}(\mathrm{x})$ in (1) by the arithmetic mean of $f\left(x_{n}\right)$ and $f\left(x_{n}^{*}\right)$ to get (4). Several authors took forward this idea of using Newton's theorem but approximating the integral $\int_{x_{n}}^{x} f^{\prime}(t) d t$ by many other variants instead of trapezoidal rule.

Özban [10] used the harmonic mean instead of arithmetic mean in (4) and obtained third order new variant of Newton's method

$$
x_{n+1}=x_{n}-\frac{f\left(x_{n}\right)\left[f^{\prime}\left(x_{n}\right)+f\left(x_{n}^{*}\right)\right]}{2 f^{\prime}\left(x_{n}\right) f^{\prime}\left(x_{n}^{*}\right)}
$$

This method is known as harmonic mean Newton's method.

Also in the same paper [10], he approximated the integral in Newton's theorem (2) by the midpoint integration rule instead of the trapezoidal rule and obtained third order variant of Newton's method

$$
x_{n+1}=x_{n}-\frac{f\left(x_{n}\right)}{f^{\prime}\left(\frac{x_{n}+x_{n}^{*}}{2}\right)},
$$


which is known as midpoint Newton's method.

Ababneh [1] used contra harmonic mean instead of arithmetic mean in (4) and obtained a third order new variant of Newton's method

$$
x_{n+1}=x_{n}-f\left(x_{n}\right) \frac{\left[f^{\prime}\left(x_{n}\right)+f^{\prime}\left(x_{n}^{*}\right)\right]}{f^{\prime 2}\left(x_{n}\right)+f^{\prime 2}\left(x_{n}^{*}\right)}
$$

Potra and Pták [11] suggested a modification of Newton's method with third order convergence defined by

$$
x_{n+1}=x_{n}-\frac{\left[f\left(x_{n}\right)+f\left(x_{n}^{*}\right)\right]}{f^{\prime}\left(x_{n}\right)}
$$

All the numerical methods mentioned above are of order three and we have to evaluate three functions per iteration in all methods.

\section{Numerical Experiments}

In this Section, we study the numerical performance of the methods which are described in previous section. Numerical computations reported here have been carried out in a Matlab software. We have used the stopping criteria $\left|x_{n+1}-x_{n}\right|<\varepsilon$, where $\varepsilon=(10)^{-12}$ and $\left|f\left(x_{n}\right)\right|<\delta$, where $\delta=(10)^{-14}$ for the iterative process of our results.

The functions, used as numerical examples are given below:
(i) $f_{1}=x^{3}+4 x^{2}-10$
(ii) $f_{2}=x^{3}-10$
(iii) $f_{3}=(x-1)^{3}-1$
(iv) $f_{4}=\cos x-x$
(v) $f_{5}=\cos x-x e^{x}+x^{2}$
(vi) $f_{6}=\sin ^{2} x-x^{2}+1$
(vii) $f_{7}=x^{2}-e^{x}-3 x+2$
(viii) $f_{8}=x^{6}-x-1$
(ix) $f_{9}=3 x+\sin x-e^{x}$

The Tables given below show the performance of the arithmetic mean Newton's method (AMNM), harmonic mean Newton's method (HMNM), midpoint Newton's method (MNM), contra harmonic mean Newton's method (CHMNM) and Potra and Pták method (PPM) when we apply these methods on each of the function mentioned above.

Table 1: $f_{1}=x^{3}+4 x^{2}-10$ and initial guess $x_{0}=3$.

\begin{tabular}{|c|c|c|c|c|}
\hline AMNM & HMNM & MNM & CHMNM & PPM \\
\hline 1.644853060442072 & 1.506854021269525 & 1.616194240542067 & 1.759501956489971 & 1.707504329878743 \\
1.369582248045579 & 1.365338826615396 & 1.367973785536972 & 1.383862438798629 & 1.376538500113823 \\
1.365230035613704 & 1.36523001341413 & 1.365230018052222 & 1.365233236336585 & 1.365230694823196 \\
1.365230013414097 & 1.365230013414097 & 1.365230013414097 & 1.365230013414097 & 1.365230013414097 \\
\hline
\end{tabular}


Table 2: $f_{2}=x^{3}-10$ and initial guess $x_{0}=3$.

\begin{tabular}{|c|c|c|c|c|}
\hline AMNM & HMNM & MNM & CHMNM & PPM \\
\hline 2.224734916017641 & 2.180912724247685 & 2.214078478002378 & 2.264106155367207 & 2.247470556468169 \\
2.154516140897244 & 2.154435380081791 & 2.154474442166916 & 2.154975247074114 & 2.154736480024608 \\
2.154434690032019 & 2.154434690031884 & 2.154434690031896 & 2.154434690105568 & 2.154434690043722 \\
2.154434690031884 & & 2.154434690031884 & 2.154434690031884 & 2.154434690031883 \\
\hline
\end{tabular}

Table 3: $f_{3}=(x-1)^{3}-1$ and initial guess $x_{0}=3$.

\begin{tabular}{|c|c|c|c|c|}
\hline AMNM & HMNM & MNM & CHMNM & PPM \\
\hline 2.223121387283237 & 2.127018454440599 & 2.200475907198096 & 2.300163497448814 & 2.263069058641976 \\
2.008192510887264 & 2.000421883962698 & 2.005099782101378 & 2.027891538551798 & 2.017370019498418 \\
2.000000630347324 & 2.000000000012531 & 2.000000120403840 & 2.000043782089048 & 2.000009899210042 \\
2.000000000000000 & 2.000000000000000 & 2.000000000000000 & 2.000000000000182 & 2.000000000000002 \\
& & & 2.000000000000000 & \\
\hline
\end{tabular}

Table 4: $f_{4}=\cos x-x$ and initial guess $x_{0}=1$.

\begin{tabular}{|c|c|c|c|c|}
\hline AMNM & HMNM & MNM & CHMNM & PPM \\
\hline 0.739058390444989 & 0.738522102659178 & 0.739939996442546 & 0.739592482894780 & 0.740087803770686 \\
0.739085133215160 & 0.739085133221149 & 0.739085133256054 & 0.739085133223504 & 0.739085133313251 \\
& 0.739085133215161 & 0.739085133215161 & 0.739085133215161 & 0.739085133215161 \\
\hline
\end{tabular}

Table 5: $f_{5}=\cos x-x e^{x}+x^{2}$ and initial guess $x_{0}=1$.

\begin{tabular}{|c|c|c|c|c|}
\hline AMNM & HMNM & MNM & CHMNM & PPM \\
\hline 0.665881945014898 & 0.649939286371225 & 0.658151439643510 & 0.639250900896522 & 0.672793782790258 \\
0.639169572742496 & 0.639154369449049 & 0.639157541165281 & 0.639154096333320 & 0.639198509615054 \\
0.639154096332011 & 0.639154096332007 & 0.639154096332008 & 0.639154096332008 & 0.639154096332115 \\
& & & & 0.639154096332007 \\
\hline
\end{tabular}

Table 6: $f_{6}=\sin ^{2} x-x^{2}+1$ and initial guess $x_{0}=2$.

\begin{tabular}{|c|c|c|c|c|}
\hline AMNM & HMNM & MNM & CHMNM & PPM \\
\hline 1.441902639265306 & 1.413080510466222 & 1.447589150284307 & 1.468026509302020 & 1.462825452363362 \\
1.404523329874331 & 1.404491677315694 & 1.404535328036127 & 1.404769406608980 & 1.404695724667290 \\
1.404491648215362 & 1.404491648215341 & 1.404491648215391 & 1.404491648242576 & 1.404491648225771 \\
1.404491648215341 & & 1.404491648215341 & 1.404491648215341 & 1.404491648215341 \\
\hline
\end{tabular}


Table 7: $f_{7}=x^{2}-e^{x}-3 x+2$ and initial guess $x_{0}=1$.

\begin{tabular}{|c|c|c|c|c|}
\hline AMNM & HMNM & MNM & CHMNM & PPM \\
\hline 0.274057949986885 & 0.274022389337211 & 0.248487682786732 & 0.274093507152986 & 0.257357237589679 \\
0.257530454327562 & 0.257530415010055 & 0.257530289365435 & 0.257530494142002 & 0.257530285439770 \\
0.257530285439861 & 0.257530285439861 & 0.257530285439861 & 0.257530285439861 & 0.257530285439861 \\
\hline
\end{tabular}

Table 8: $f_{8}=x^{6}-x-1$ and initial guess $x_{0}=1$.

\begin{tabular}{|c|c|c|c|c|}
\hline AMNM & HMNM & MNM & CHMNM & PPM \\
\hline 1.105652850091284 & 1.135893960625761 & 1.115432653127186 & 1.086421152078458 & 1.042803200000000 \\
1.134522904945114 & 1.134724140685710 & 1.134684773560854 & 1.132876348819721 & 1.115915643113079 \\
1.134724138342278 & 1.134724138401519 & 1.134724138401206 & 1.134724054935787 & 1.134635489540452 \\
1.134724138401519 & & 1.134724138401519 & 1.134724138401519 & 1.134724138393373 \\
& & & & 1.134724138401520 \\
\hline
\end{tabular}

Table 9: $f_{9}=3 x+\sin x-e^{x} \quad$ and initial guess $\quad x_{0}=2.5$.

\begin{tabular}{|c|c|c|c|c|}
\hline AMNM & HMNM & MNM & CHMNM & PPM \\
\hline 1.975501614079307 & 1.930129220588023 & 1.962057585618187 & 2.014181843620479 & 1.995583833798580 \\
1.890585083862651 & 1.890039622289577 & 1.890308114900232 & 1.892816236734752 & 1.891602591276880 \\
1.890029729433217 & 1.890029729251985 & 1.890029729270506 & 1.890029771899502 & 1.890029736418303 \\
1.890029729251985 & & 1.890029729251985 & 1.890029729251985 & 1.890029729251985 \\
\hline
\end{tabular}

\section{Conclusion}

In Section 3, if we observe carefully in all Tables, harmonic mean Newton's method converges faster than all other mentioned methods and Potra and Pták method has less degree of convergence than all other mentioned methods in most of the cases for mentioned error tolerance. However, in case of function $f_{4}$, all other methods take three iterations to get the simple solution but arithmetic mean Newton's method, i.e., trapezoidal method takes only two iterations for the supposed initial guess. Also from Table 7, for the function $f_{7}$, Potra and Pták method converges faster than all other mentioned methods. Thus, in general, it is clear that iterative methods having the same order of convergence and the same efficiency index may not perform equally for a given nonlinear equation of a single variable. 


\section{References}

[1] Ababneh OY (2012), New Newton's method with third order convergence for solving nonlinear equations, World Academy of Science and Engineering and Technology, 61:10711073.

[2] Bradie B (2007), A friendly introduction to Numerical Analysis, Pearson.

[3] Burdan LR and Fairs JD (2011), Numerical Analysis, Cengage Learning.

[4] Dheghain M and Hajarian M (2010), New iterative method for solving nonlinear equations fourth-order convergence, International Journal of Computer Mathematics, 87: 834-83.

[5] Frontini M and Sormani E (2003), Some variant of Newton's method with third-order convergence,

Applied Mathematics and Computation, 140:419-426

[6] Jain D (2013), Families of Newton-like methods with fourth-order convergence, International Journal of computer mathematics, 90: 1072-1082.

[7] Jain P, Bhatta CR and Jnawali J (2015), Modified Newton type methods with higher order convergence, Jordan Journal of Mathematics and Statistics, 8(4):327-341.

[8] Kou J, Li Y, and Wang X (2006), A modification of Newton's method with third-order convergence, Applied Mathematics and Computation 181:1106-1111.

[9] McDougall TJ, Weatherspoon SJ (2014), A simple modification of Newton's method to achieve Convergence of order $1+\sqrt{2}$, Applied Mathematics Letters 29: 20-25

[10] Özban AY (2004), Some new variants of Newton's method, Applied Mathematics Letters, 13: 677-682.

[11] Potra FA and Pták V (1984), Non-discrete induction and iterative Processes, Research Note in Mathematics, 203, Pitman, Boston.

[12] Wang P (2011), A third-order family of Newton-like iteration method for solving nonlinear Equations, Journal of Numerical Mathematics and Stochastics, 3(1): 13-19

[13] Weerakoon S and Fernando TGI (2002), A variant of Newton's method with accelerated thirdorder convergence, Applied Mathematics Letters 13: 87-93. 